\title{
Ein neuer Ansatz für eine präzise automatische Temperaturkalibrierung von Microhotplates
}

\author{
Robin Diener ${ }^{1}$, Caroline Schultealbert ${ }^{1}$, Tobias Baur ${ }^{1}$, Andreas Schütze $^{1}$, Tilman Sauerwald ${ }^{1}$ \\ ${ }^{1}$ Lehrstuhl für Messtechnik, Universität des Saarlandes, Campus A5 1, 66123 Saarbrücken, \\ Deutschland \\ E-Mail: c.schultealbert@Imt.uni-saarland.de
}

\section{Zusammenfassung}

In dieser Arbeit wird eine Methode für die Temperaturkalibrierung von Microhotplates am Beispiel von MEMS-Gassensoren vorgestellt. Als Bezugspunkte dienen Fest-Flüssig-Phasenübergänge von Referenzmaterialien. Die latente Wärme des Phasenübergangs führt zu einem Anstieg der Wärmekapazität, was sich bei einer definierten Heizrampe in einem lokalen Maximum der Heizleistung widerspiegelt. Die Methode wird mit einem AS-MLV-P2 Gassensor (ams Sensor Germany $\mathrm{GmbH}$ ) mit integriertem Membranheizer getestet. Als Referenzmaterial für die Entwicklung der Methode wird Hexatriacontan verwendet, dessen Schmelzpunkt bei $76{ }^{\circ} \mathrm{C}$ als erster und die Raumtemperatur $\left(22^{\circ} \mathrm{C}\right)$ als zweiter Kalibrierpunktpunkt dient. Durch den hohen Siedepunkt von $497^{\circ} \mathrm{C}$ kann die Reproduzierbarkeit des Verfahrens getestet werden. Der Schmelzpunkt kann als Onset-Punkt des Schmelzvorgangs mit einer Wiederholgenauigkeit von $\pm 0,15 \mathrm{~K}$ ermittelt werden. Bei variierenden Versuchsbedingungen (Heizrate) liegt die Wiederholgenauigkeit weiterhin deutlich unter $1 \mathrm{~K}$. Weitere Versuche werden mit den Referenzmaterialien Pyrazin und Urotropin durchgeführt, da diese durch einen relativ niedrigen Siedepunkt von $\left(115^{\circ} \mathrm{C}\right.$ und $\left.263^{\circ} \mathrm{C}\right)$ und ihre gute Löslichkeit in Wasser und Alkoholen auch innerhalb von Fertigungsprozessen reversibel appliziert und entfernt werden können. Bei beiden Materialien konnten die Phasenübergangstemperaturen aus dem Heizleistungsverlauf präzise bestimmt werden.

Keywords: Temperaturkalibrierung, MEMS Sensoren, Gassensorik, Microhotplate, Phasenübergang

\begin{abstract}
Motivation
Die präzise Messung und Steuerung der Temperatur von Microhotplates ist oft fehlerbehaftet. Für eine Messung der Temperatur nach dem Stefan-BoltzmannGesetz sind die Emissionskoeffizienten und die Transparenz der Membranen häufig nicht tabelliert [1] und auch experimentell sehr schwer zu bestimmen. Bei einer Kalibrierung über den Temperaturkoeffizienten des Heizerwiderstands wird das gesamte Bauteil (z.B. in einer Klimakammer) erhitzt. Dabei wirkt sich der Temperaturkoeffizient auch auf die signifikanten Zuleitungswiderstände aus, die außerhalb der geheizten Membran liegen und daher im Regelbetrieb nicht erwärmt werden. Allerdings sind für viele MEMS-Anwendungen exakte Messung und Regelung der Temperatur notwendig. Ein Beispiel sind Halbleitergassensoren, bei denen viele Prozesse (z.B. die thermisch aktivierte Leitfähigkeit und stattfindende Oberflächenreaktionen mit Aktivierungsenergie) stark temperaturabhängig sind [2]. Insbesondere für die Übertragbarkeit
\end{abstract}

$\begin{array}{ll}\text { von Kalibriermodellen ist } \begin{array}{r}\text { eine } \\ \text { sensorübergreifende }\end{array} & \text { korrekte }\end{array}$ Temperatureinstellung absolute Notwendigkeit. Derzeit wird hierfür am Lehrstuhl für Messtechnik die meist vorhandene LeistungsTemperatur-Kennlinie (oft lediglich durch einen Arbeitspunkt im Datenblatt gegeben) in eine gemessene Widerstands-TemperaturKennlinie überführt, sodass der Heizerwiderstand als Regelgröße verwendet werden kann [3].

In dieser Arbeit wird deshalb eine Methode für die Temperaturkalibrierung von Microhotplates am Beispiel von MEMS-Gassensoren vorgestellt, die sich sowohl in der Herstellung als auch im Nachgang, beispielsweise beim Anwender, auf jedes Einzelelement anwenden lassen. Die Kalibrierroutine muss daher sowohl einfach und kostengünstig als auch zerstörungsfrei sein. Als Bezugspunkte dienen Fest-Flüssig-Phasenübergänge von Referenzmaterialien, was ein in der Praxis anerkanntes Verfahren zur Temperaturkalibrierung darstellt [4].

Die latente Wärme des Phasenübergangs führt zu einem temporären Anstieg der 
Wärmekapazität, was sich bei einer definierten Heizrampe in einem lokalen Maximum der Heizleistung widerspiegelt. Ziel ist es, ein reproduzierbares Merkmal dieses Maximums zu finden, das mit der normalen Betriebselektronik dieser Sensoren vermessen und ausgewertet werden kann. Darüber hinaus werden Materialien gesucht, die einerseits den interessanten Temperaturbereich des Sensors mit ihrer Phasenübergangstemperatur abbilden und andererseits leicht aufzubringen sowie zerstörungsfrei zu entfernen sind, hierfür sind insbesondere sublimierende Materialien von Interesse.

\section{Experimenteller Aufbau und Methoden}

Die Methode wird mit einem AS-MLV-P2 Gassensor (ams Sensor Germany $\mathrm{GmbH}$ ) mit integriertem Membranheizer aus Platin getestet. Der Widerstand der Heizerschicht dient als Führungsgröße der Regelung. Aufgrund des Temperaturkoeffizienten von Platin lässt sich (bei gleichbleibender Temperatur der Zuleitungswiderstände) der Heizerwiderstand eindeutig mit einer Membrantemperatur identifizieren. Der elektronische Aufbau der Heizerregelung ist identisch zu der Elektronik, die am Lehrstuhl für Messtechnik (LMT) für die Vermessung von Gassensoren im temperaturzyklischen Betrieb (engl. temperature cycled operation, TCO) verwendet wird [5]. Sie beinhaltet eine Vierleitermessung, um Zuleitungswiderstände außerhalb des Sensors zu unterdrücken. Um bei der Raumtemperaturmessung Eigenerwärmung zu verhindern, wird der Heizerwiderstand mit einem Stromfluss von unter $1 \mu \mathrm{A}$ ermittelt. Dieser Widerstand kann daher als zweiter Kalibrierpunkt zur Ermittlung einer Widerstands-Temperatur-Kennlinie verwendet werden.

Für die Methodenentwicklung wird Hexatriacontan als Referenzmaterial verwendet (Schmelzpunkt $74-76^{\circ} \mathrm{C}$ [6]), da es aufgrund des geringen Dampfdrucks nach einer Applikation mehrmals reproduzierbar vermessen werden kann. Ein aufgebrachter möglichst kleiner Flake des Materials wird zunächst aufgeschmolzen, sodass sich eine homogene Schicht auf der Hotplate bildet, diese Schicht kann dann mehrmals für die Messung aufgeschmolzen werden.

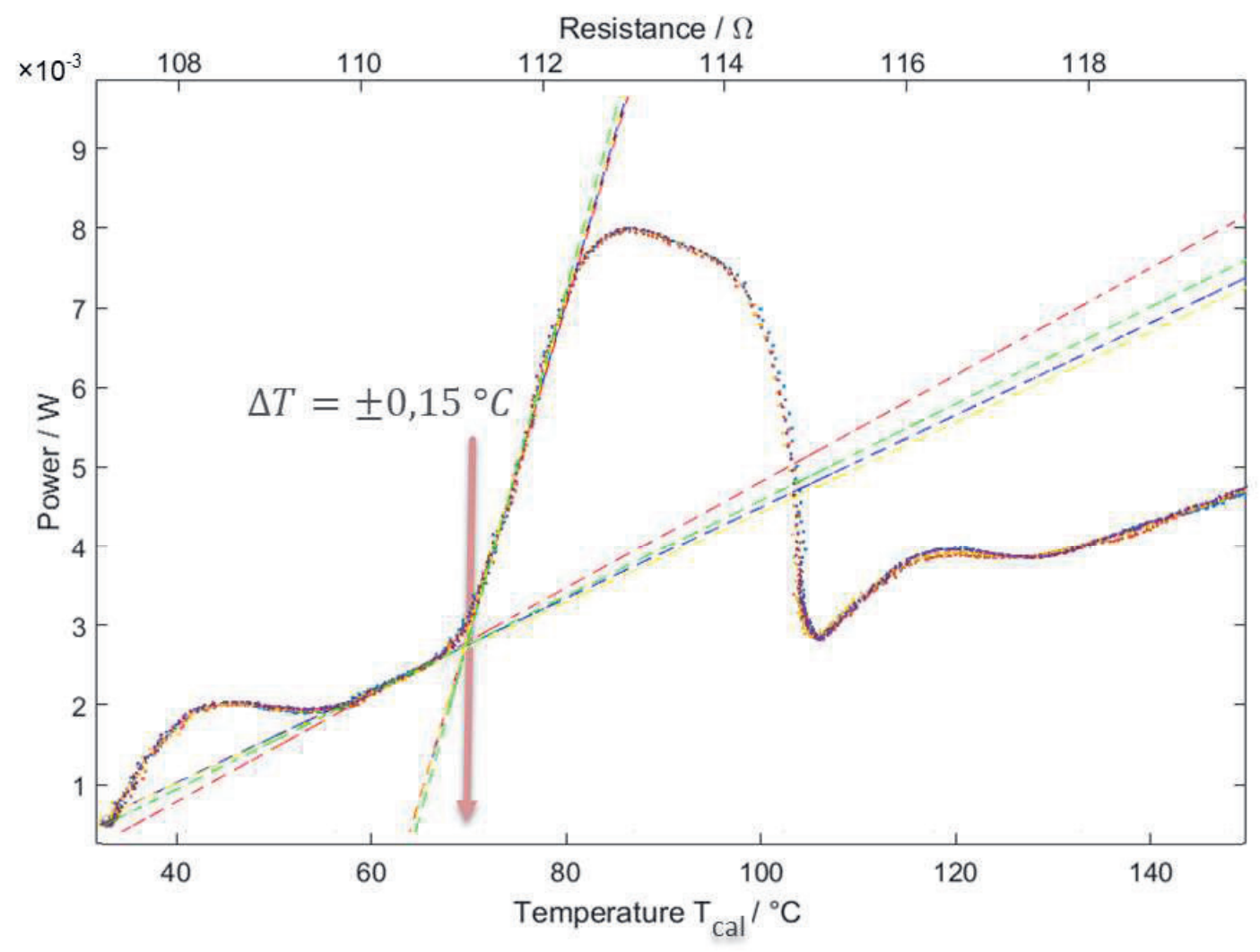

Abb. 1: Mehrmalige Temperaturkalibrierung über die Heizleistung mit derselben HexatriacontanSchicht auf der Microhotplate. Die bestimmten Onset-Punkte der Kurven liegen maximal $0,15^{\circ} \mathrm{C}$ auseinander. 
Als weitere Referenzmaterialien werden Pyrazin (Schmelzpunkt ca. $56^{\circ} \mathrm{C}$, Siedepunkt $115-116^{\circ} \mathrm{C}$ [7]) und Urotropin (Sublimationspunkt $263{ }^{\circ} \mathrm{C}$ [8]) verwendet. Beide Substanzen können in Wasser oder (in geringerer Konzentration) in Methanol gelöst und dann auf die Hotplate aufgetropft werden.

Für unsere Untersuchungen wurde das Pyrazin als Feststoff (Flake) aufgebracht und das Urotropin als Lösung in Wasser oder Methanol. Das Lösemittel muss bei Raumtemperatur verdunstet werden, bevor der eigentliche Kalibriervorgang startet. Ein Erwärmen der Hotplate, die diese Verdunstung beschleunigt, kann dazu führen, dass die Urotropin-Schicht mit dem entstehenden Gas von der Hotplate abgelöst wird, wodurch die Schicht ohne Phasenübergang von der Hotplate entfernt wird (vermutlich als Partikel im Gasstrom). Für die in Abb. 3 gezeigte Messung wurde eine gesättigte UrotropinMethanol Lösung verwendet.

\section{Ergebnisse}

In Abb. 1 sind mehrere Schmelzkurven derselben Hexatriacontan-Schicht dargestellt. Aufgetragen ist die benötigte Heizleistung über dem Heizerwiderstand (obere x-Achse). Diese kann nach der Temperaturkalibrierung direkt in eine Temperatur überführt werden (untere $\mathrm{x}$ Achse). Angenommen wird hierbei ein linearer Zusammenhang. Die Heizrate entspricht auf Basis der in der Einleitung beschriebenen vorläufigen Temperaturkalibrierung [2] etwa $80 \mathrm{~K} / \mathrm{s}$ im Temperaturbereich von $30^{\circ} \mathrm{C}$ bis $150^{\circ} \mathrm{C}$, geregelt wird auf den entsprechenden Heizerwiderstand.

Die Bestimmung der Schmelztemperatur erfolgt am Onset-Punkt des Heizleistungspeaks, der den Beginn des Phasenübergangs definiert. Bestimmt wird der Onset-Punkt als Schnittpunkt zweier Regressionsgeraden der Heizleistung in Abhängigkeit des Heizerwiderstands. Die erste Gerade wird im linearen Bereich des Aufheizvorgangs nach dem Einschwingen der Regelung und vor dem Auftreten des Schmelzpeaks gefittet. Die zweite Regressionsgerade wird durch den Anstieg des Schmelz-Peaks gelegt. Der Onset-Punkt kann auf diese Art und Weise für vier Messungen mit einer Wiederholgenauigkeit von $\pm 0,15{ }^{\circ} \mathrm{C}$ ermittelt werden.

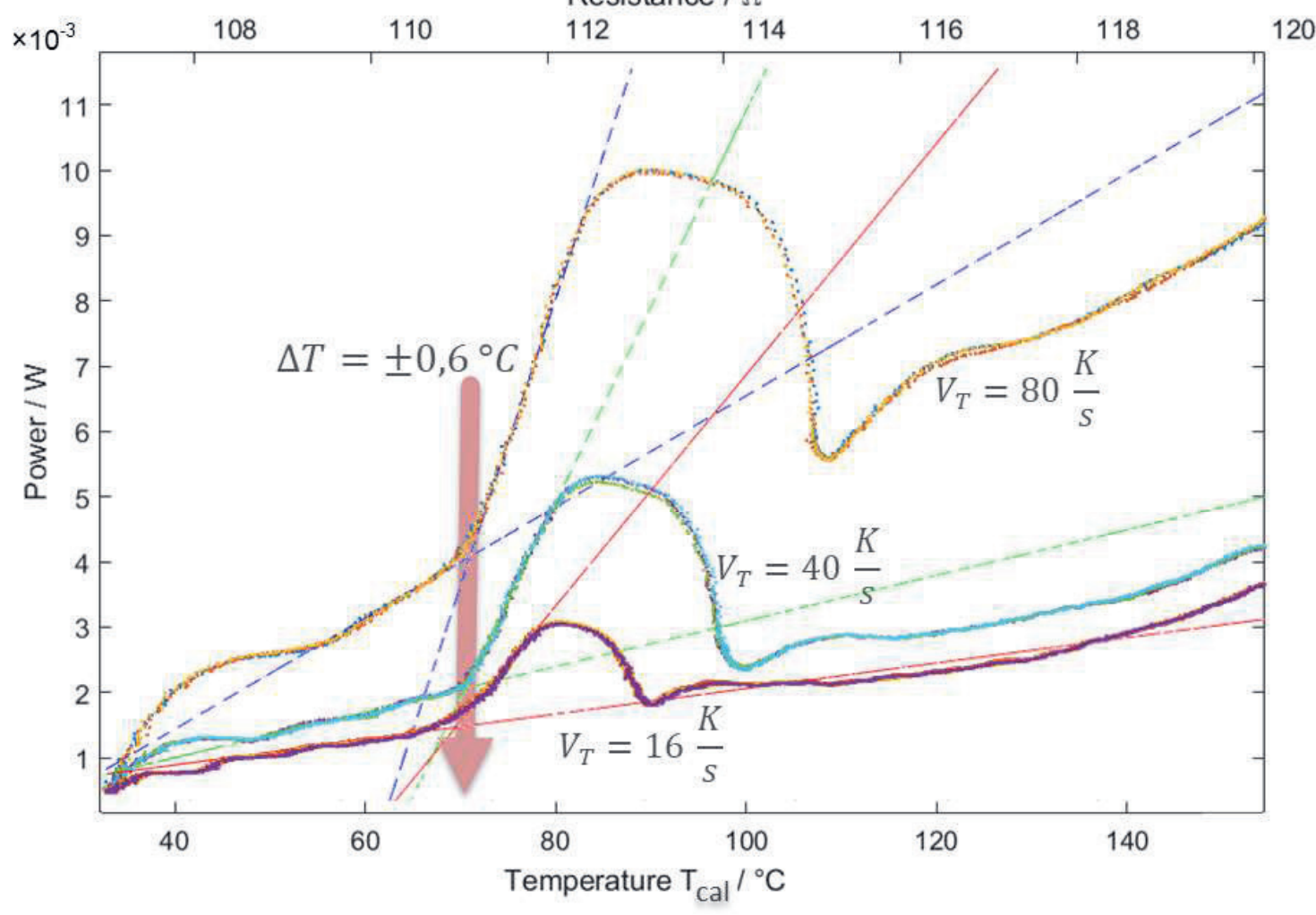

Abb. 2: Auch bei Veränderung der Heizrate, die stellvertretend für andere Einflüsse wie unterschiedliche Mengen Referenzmaterial herangezogen wurde, kann die Bestimmung des Onset-Punktes des Schmelzprozesses auf $\pm 0,6^{\circ} \mathrm{C}$ genau erfolgen. Die Verwendung anderer Charakteristika des Schmelzpeaks (z.B. Maximum) sind deutlich weniger erfolgsversprechend, da sie sich durch die Heizratenvariation verschieben. 
Abb. 2 zeigt die Vermessung der Hexatriacontan-Schicht bei drei unterschiedlichen Heizraten $\mathrm{v}_{\mathrm{T}}$ : $16 \mathrm{~K} / \mathrm{s}, 40 \mathrm{~K} / \mathrm{s}$ und $80 \mathrm{~K} / \mathrm{s}$, Die Auftragung ist analog zu Abb. 1. Durch diese Variation lässt sich abschätzen, wie sich eine Variation der Stoffmenge an aufgebrachtem Phasenwechselmaterial auf die Genauigkeit der Kalibrierung auswirkt. Wir gehen davon aus, dass beide Variationen vergleichbare Fehler hervorrufen, da die Heizleistungen über den gesamten Zeitverlauf der Messung gleich skalieren. Die maximale Abweichung der bestimmten Onset-Punkte beträgt trotz Variation der Heizrate um den Faktor 5 nur $\pm 0,6{ }^{\circ} \mathrm{C}$. Die sehr reproduzierbare Bestimmung der Temperatur bei unterschiedlichen Heizraten lässt auf eine geringe Anfälligkeit für Fehler besonders auch in Bezug auf die aufgebrachte Stoffmenge schließen. In Abb. 2 wird auch deutlich, dass eine Bestimmung anderer Parameter des aufgenommenen Schmelzpeaks, wie beispielsweise das Maximum, keine reproduzierbaren Ergebnisse für die Temperaturkalibrierung liefern, da sie sich in Abhängigkeit von der Heizrate verschieben. Das liegt vermutlich daran, dass der Phasenübergang nicht im gesamten Material homogen erfolgt und dann weitere Parameter wie z.B. Wärmetransport in der Hexatriacontanschicht eine Rolle spielen.

Die Verwendung von Hexatriacontan als Referenzmaterial dient, wie bereits erwähnt, zur Methodenentwicklung, da das Material durch den geringen Dampfdruck vollständig auf dem Heizer verbleibt und die Messungen wiederholbar sind. Eine rückstandsfreie Entfernung des Schmelzmaterials kann bei Temperaturen über $500{ }^{\circ} \mathrm{C}$ erfolgen. Eine Beeinflussung der Sensorschicht kann dabei jedoch nicht ausgeschlossen werden. Die Aufbringung von wachsartigen Referenzmaterialien wie Hexatriacontan ist daher nicht für eine zerstörungsfreie Kalibrierung geeignet.

Für eine rückstandsfreie Kalibrierung können Referenzmaterialien mit höherem Dampfdruck bzw. niedrigem Siedepunkt verwendet werden, die, bereits kurz nach dem schmelzen direkt in die Dampfphase übergehen. Ein Beispiel ist Pyrazin, welches beim Erhitzen bereits vollständig verdampft, noch bevor der Siedepunkt erreicht ist.

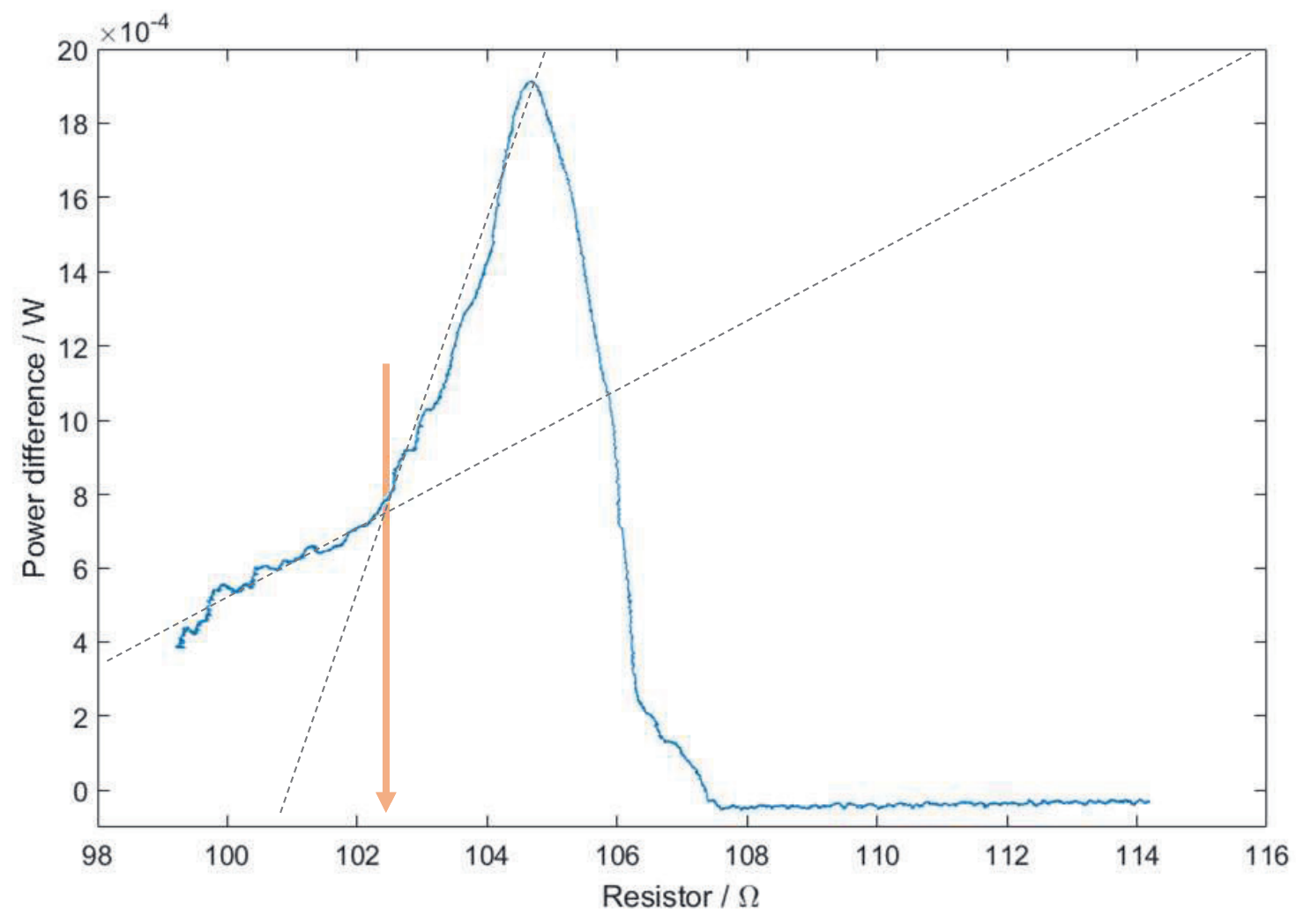

Abb. 3: Kalibrierung der Temperatur über die Bestimmung des Onset-Punkts des Schmelz- und Verdampfungsprozesses von Pyrazin, welches für die automatisierte und fertigungsnahe Kalibrierung besser geeignet ist. 


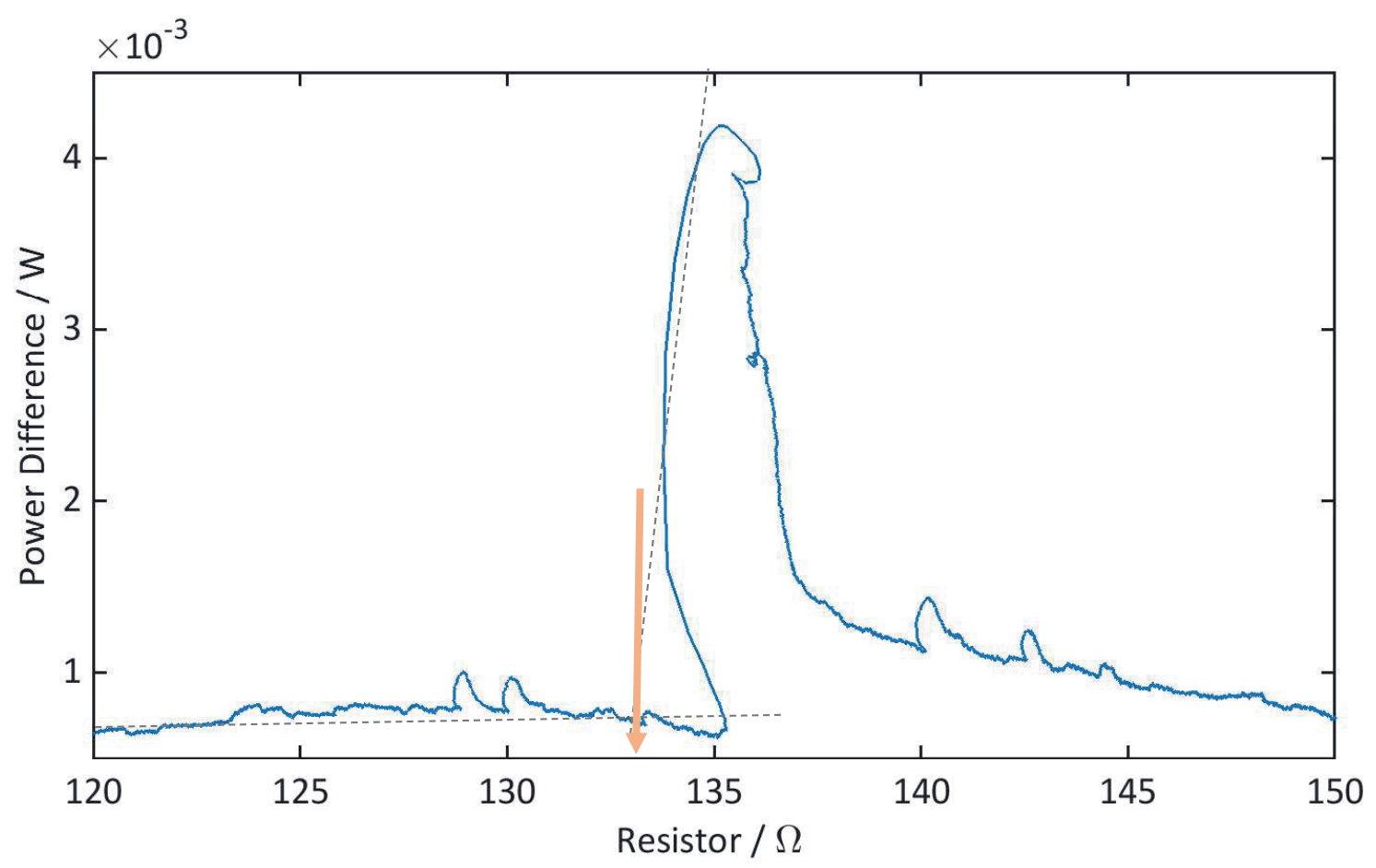

Abb. 4: Kalibrierung der Temperatur über den Sublimationsprozesses von Urotropin, welches aus der Flüssigphase aufgebracht werden kann und dessen Phasenübergang in einem für Gassensoren geeigneteren Temperaturbereich liegt. Die Heizrate und Menge des aufgebrachten Materials sollten noch weiter optimiert werden, um ein reproduzierbares Merkmal, wie den Onset-Punkt bestimmen zu können.

Abb. 3 zeigt eine Heizleistungskurve mit einem aufgebrachten Pyrazin-Flake auf der Schicht, wobei die Basislinie des leeren Heizers abgezogen wurde. Auch hier ist der OnsetPunkt des Schmelzprozesses problemlos zu bestimmen. Nach dem Maximum der Heizleistung liegt die Differenz zwischen dem leeren Heizer und dieser Messung nahezu bei $0 \mathrm{~W}$, sprich es verbleibt kein Material auf dem Heizer, da dieses sich in Form einer erhöhten Wärmekapazität und einer damit erhöhten Heizleistung bemerkbar machen würde (vgl. Kurve vor dem Phasenübergang).

Weitere Versuche mit Pyrazin wurden nicht durchgeführt, da der Temperaturbereich zumindest für Halbleitergassensoren nicht optimal ist. Stattdessen wurden Versuche mit Urotropin durchgeführt, da dieses Material aus der Flüssigphase auf der Microhotplate abgeschieden und anschließend rückstandsfrei sublimiert bzw. zersetzt werden kann. Die Zersetzungsprodukte sind sehr leicht flüchtig, daher ist es naheliegend, dass diese nicht auf der Sensorschicht verbleiben und sie langfristig beeinflussen: Kohlenmonoxid, Kohlendioxid, Stickoxide, Ammoniak und Formaldehyd [8]. Der Sublimationspunkt von Urotropin liegt deutlich besser im Betriebsbereich von Halbleitergassensoren im TCO.
Für die Messung wurde ein Tropfen gesättigte Urotropin-Methanol-Lösung direkt auf der Hotplate platziert. Nach mehreren Stunden an Raumluft ist das Lösungsmittel komplett verdampft und lediglich ein Urotropin-Flake bleibt zurück (ca. $100 \mathrm{nl}$ ). Für den Sublimationsprozess wurde die Hotplate bei einer Heizrate von $12 \mathrm{~K} / \mathrm{s}$ von $50{ }^{\circ} \mathrm{C}$ auf $350{ }^{\circ} \mathrm{C}$ aufgeheizt. Der Verlauf der Heizleistung (mit abgezogener Basislinie) ist in Abb. 4 dargestellt. Materialmenge, Heizrate und Regelungsparameter sind bei dieser Messung noch nicht optimal abgestimmt, da die Hotplate ein Temperaturmaximum durchläuft bevor sie durch den Sublimationsprozess wieder abgekühlt wird. Für eine eindeutige Bestimmung des OnsetPunktes und damit eine reproduzierbare Temperaturkalibrierung der Hotplate, müssen diese Parameter noch optimiert werden.

\section{Zusammenfassung und Ausblick}

Mit den hier gezeigten Messergebnissen konnte gezeigt werden, dass eine Temperaturkalibrierung von Microhotplates mit Genauigkeiten unter $1 \mathrm{~K}$ mit Hilfe von Phasenübergängen (Schmelzen, Sublimieren) möglich ist. Die sehr gute Reproduzierbarkeit kann mit schwerflüchtigen Substanzen wie Hexatriacontan gezeigt werden. Für die 
Anwendung im Fertigungsprozess sind Substanzen, die aus der Flüssig abgeschieden werden können, wünschenswert, was hier am Beispiel von Pyrazin und Urotropin gezeigt wurde. Ein Nachweis der Reproduzierbarkeit ist hierbei schwieriger. Weitere Untersuchungen zur Optimierung der ProzessParameter und Wiederholgenauigkeit der Messungen sind hier noch notwendig. Die vorgestellte Methode ermöglicht die zerstörungsfreie Kalibrierung von MEMS Gassensoren, bei denen die exakte Temperaturkalibrierung jedes einzelnen Sensors aufgrund von Fertigungsschwankungen eine enorme Verbesserung für die Übertragbarkeit von Kennlinien von verschiedenen Sensoren des gleichen Typs bedeutet.

\section{Literaturnachweis}

[1] R. Zhang, Y. Gu, D. Zhu, J. Hao, Z. Tang, Thermal measurement and analysis of micro hotplate array using thermography, Sensors and Actuators A: Physical. 2002, 100, 144-152, doi: 10.1016/S0924-4247(02)00061-4

[2] G. Korotcenkov, B. K. Cho, Instability of metal oxide-based conductometric gas sensors and approaches to stability improvement (short survey), Sensors and Actuators B: Chemical. 2011, 156, 527-538, doi:10.1016/j.snb.2011.02.024.

[3] C. Schultealbert, T. Baur, A. Schütze, T. Sauerwald, Facile Quantification and Identification Techniques for Reducing Gases over a Wide Concentration Range Using a MOS Sensor in Temperature-Cycled Operation, Sensors, 2018, 18(3), 744, doi:10.3390/s18030744

[4] S. M. Sarge, E. Gmelin, G. W. H. Höhne, H. K. Cammenga, W. Hemminger, and W. Eysel, The caloric calibration of scanning calorimeters, Thermochimica Acta, 1994, 247, 2, 129-168, doi:10.1016/0040-6031(94)80118-5

[5] T. Baur, C. Schultealbert, A. Schütze, T. Sauerwald, Devicce for the detection of short trace gas pulses, $\mathrm{tm}-$ Technisches Messen, 2018, 85, doi: 10.1515/teme-2017-0137

[6] Thermo Fisher $\mathrm{GmbH}$, Hexatriacontane SDS, 2018. Online verfügbar: https://www.alfa.com/de/msds/?language=DE\& subformat $=$ CLP1\&sku=31641, aufgerufen am 29.04.2019.

[7] Thermo Fisher GmbH, Pyrazine SDS, 2018. Online verfügbar: https://www.alfa.com/de/msds/?language=DE\& subformat=CLP1\&sku=16326, aufgerufen am 29.04.2019.

[8] Thermo Fisher $\mathrm{GmbH}$, Hexamethylenetetramine SDS, 2018. Online verfügbar: https://www.alfa.com/de/msds/?language=DE\& subformat $=$ CLP1\&sku=36462, aufgerufen am 29.04.2019. 